\title{
Macroscopic-microscopic approach to the nuclear fission process
}

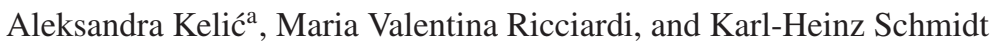 \\ GSI, Planckstr. 1, 64291 Darmstadt, Germany

\begin{abstract}
A model based on the macroscopic-microscopic approach for calculating the fission-fragment properties is presented. Using this model, a large set of experimental data measured in low- and high-energy fission can be
\end{abstract} \\ reproduced using one and same set of model parameters.
}

\section{Introduction}

Corresponding to a large-scale collective motion where both static and dynamic properties of nuclear matter play a crucial role, nuclear fission represents an excellent tool for studying different characteristics of nuclear matter, e.g., nuclearstructure effects at large deformations [1-3], dissipative phenomena like diffusion and friction in nuclei $[4,5]$ or multiphonon giant resonances [6]. Knowledge on nuclear fission is also essential for large range of applications such as the r-process nucleosynthesis [7], production of neutron-rich radioactive beams and super-heavy elements, design of neutron spallation sources and accelerator-driven systems [8], new generation of nuclear power plants [9], or nuclear safety and security $[10,11]$.

One of the important aspects of the nuclear fission process needed to answer the above questions is the fragment formation in fission, i.e., how the mass, nuclear charge and excitation energy are attributed to the nascent fragments. This issue is especially challenging at low excitation energies where shell effects and pairing correlations have considerable influence on the mass- and element-distributions of fission fragments.

Most model descriptions of the fission process follow one of the following approaches: either the evolution of the fissioning system is described with a purely theoretical model, see, e.g., [12-14] or the measured observables - mass and nuclear charge distributions and kinetic energies - are fitted by suitable functions with empirically determined parameters $[15,16]$. The first approach is very challenging: due to the complexity of the problem, any theoretical model has to introduce a certain level of simplifications. In addition, one has to face the problem that the theoretical models are able to predict the relevant properties of a nuclear system only with a limited accuracy. This is obvious for the potentialenergy surface in deformation space. Even in the nuclear ground-state configuration, where the single-particle structure is generally very well studied, the measured binding energies can only be reproduced with a standard deviation in the

${ }^{a}$ Presenting author, e-mail: a.kelic@gsi.de order of half an MeV. Such deviations are crucial for fission, e.g., a shift of $500 \mathrm{keV}$ in the ground-state binding energy modifies the spontaneous-fission half life by about 2 orders of magnitude [17]. Similar or even larger uncertainties are expected in the competition between different fission paths in low-energy fission. Thus, these models are very important for improving our understanding of the fission process, but their ability for quantitative predictions seems to be still rather limited. Moreover, in some cases they are very time consuming which limits their use for applications.

Following the second approach, one is able to reproduce existing data very well. However, the predictive power of phenomenological models for extrapolations far from presently explored regions is rather low due to the lack of the essential physics. In order to surmount these problems, we have developed a model, which combines features of these two approaches. This model is the subject of the present contribution. A preliminary version of the model was described in refs. $[18,19]$.

\section{Model description}

The semi-empirical model for the prediction of the nuclide distribution in fission is imbedded in the dynamic de-excitation code ABLA07 [20,21], which considers the competition between emission of gammas, neutrons, light charged particles $(Z \leq 2)$ and intermediate-mass fragments on one side and fission on the other side. For excitation energies above the corresponding threshold also break-up and the simultaneous emission of several fragments is considered [22]. Fission is treated as a dynamical process, taking into account the role of dissipation in establishing quasi-equilibrium in the quasibound region by the implementation of a time-dependent fission-decay width [23] with a proper description of the initial conditions included [24]. When the system passes the fission barrier and proceeds to fission, it is characterised by mass and atomic number, excitation energy and angular momentum. In the model, probabilities to evaporate neutrons and light charged particles on the descent from saddle to scission are calculated, and the probability that the system ends up in one of the many possible configurations characterized by two 
fission fragments with atomic numbers $Z_{1,2}$, mass numbers $A_{1,2}$, kinetic energies $E_{1,2}^{k i n}$, excitation energy $E_{1,2}^{e x c}$ is predicted. After two fission fragments are formed, their deexcitation is followed until their excitation energies fall below the lowest particle-emission threshold.

\subsection{Basic assumptions}

Since the available phase space is a very important driving force of any process in nature, we consider the statistical model as the basis of our model. In other words, the probability that a system is found in a certain configuration is determined by the available phase space above the corresponding point on the potential-energy surface. In order to avoid the above-mentioned uncertainties in theoretical calculations of the potential-energy surface in deformation space, we have decided to parameterize the nuclear potential as a function of the mass-asymmetry degree of freedom. This parameterization is based on the macroscopic-microscopic approach [12,2527], and we assume that the fission-fragment mass distributions can be explained by macroscopic and microscopic properties of the potential-energy landscape. The macroscopic properties of the potential-energy landscape of the fissioning system are attributed to the strongly deformed fissioning system, which are deduced from mass distributions at high excitation energy [28] and Langevin calculations [29]. The microscopic properties of the potential-energy landscape of the fissioning system are given by the qualitative features of the shell structure in the nascent fragments. Their formulation is guided by shell-model calculations [25-27]. This way, the macroscopic and the microscopic properties are strongly separated, and the number of free parameters is independent from the number of systems considered. On the other hand, the fission process is characterized also by the friction force, which slows down the directed motion towards scission. Therefore, apart form the statistical arguments one must carefully consider also dynamical aspects of the motion from saddle to scission. In several fission models based on the statistical model, e.g., [14,30,31], it was avoided to consider dynamical effects by applying the statistical model either at the saddle or at the scission configuration. This is a rather severe simplification, since, depending on the relaxation times of the different collective degrees of freedom, some influence of the motion from saddle to scission can be present. Therefore, we will discuss this point with some care.

\subsubsection{High-energy fission}

First we start considering fission at high excitation energies, where shell effects and pairing correlations are negligible. Measured mass distributions of heavy fissioning nuclei above the Businaro-Gallone point (i.e., $Z^{2} / A>22$ ) from high excitation energies can well be described by a Gaussian distribution. This finding is related to the available number of states above the potential energy as a function of mass asymmetry [32]. The second derivative $c_{A}=\mathrm{d}^{2} U /\left(\mathrm{d} A_{1}\right)^{2}$ of the potential as a function of the mass of one of the nascent fragments is related to the standard deviation $\sigma_{A}$ of the mass distribution by the following relation:

$$
\sigma_{A}^{2}=\frac{T}{2 \cdot c_{A}} .
$$

Where $T$ is the nuclear temperature, which is related to the excitation energy of the fissioning system $E=a \cdot T^{2}$. The coefficient $a$ is the level-density parameter. This relation is a very important ingredient of the macroscopic part of our model. Firstly, there exists a large body of experimental data on $\sigma_{A}$ values, which provides the empirical data basis for a realistic prediction of fission mass distributions when structural effects are negligible. Secondly, the empirical result that the variance $\sigma_{A}^{2}$ is proportional to the nuclear temperature supports the validity of the statistical model. However, it is difficult to extract from these data, at which moment on the descent from saddle to scission the decision on the width of the mass distribution is taken. We can consider two possible extremes: in one case, the phase space near the saddle point determines the mass asymmetry of the system, which is more or less frozen on a fast descent to scission. In the other case, the mass asymmetry degree of freedom adjusts very fast to the potential and is, therefore, finally determined close to scission. Since a variation of the mass asymmetry is connected with a substantial transport of nucleons and, consequently, the inertia of this collective degree of freedom should be large, we tend to support the first possibility. Following this idea, we take the systematics established in ref. [32] using the temperature at saddle in equation (1) for deducing the second derivative $c_{A}$ of the potential from the experimental data. Thus, we have the first quantitative relation, which we use in our model to calculate the width of the mass distribution in case of sufficiently high excitation energies. One example of mass distribution in the spallation of ${ }^{238} \mathrm{U}$ by $1 \mathrm{GeV}$ proton is shown in figure 1.

On the other hand, the $N / Z$ collective degree of freedom can be considered as a fast degree of freedom, as it is enough to exchange very few neutrons or protons between the two nascent fragments in order to explore the full $N / Z$ range observed in the final fragments. Therefore, we assume that the $N / Z$ degree of freedom is determined, opposite to mass asymmetry, near the scission point, and we calculate its value taking into account the number of evaporated particles between the saddle and the scission point as well as the charge-polarisation effect governed by the liquid-drop contribution to the energy at the scission point [34].

\subsubsection{Low-energy fission}

Considering the fission process at lower excitation energies, our approach has to be substantially extended in order to include the appearance of fission channels. Early ideas for this concept are formulated in ref. [35].

Following the hypothesis that the mass-asymmetry degree of freedom is essentially frozen on the way from saddle to scission, the probabilities for the population of the different fission channels should be decided near the outer saddle. Therefore, we assume that there is a direct correspondence between the shape of the potential near the outer saddle as a function of the mass asymmetry and the population of the fission channels. 


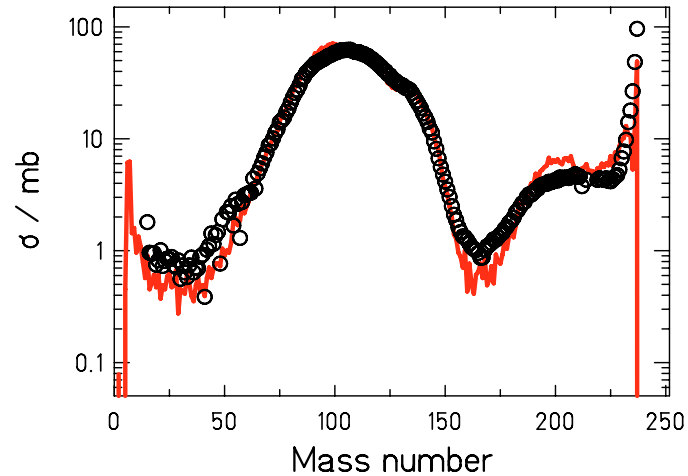

Fig. 1. Calculated mass distribution (full line) for the reaction ${ }^{1} \mathrm{H}$ $(1 \mathrm{GeV})+{ }^{238} \mathrm{U}$ compared to data measured at GSI $[20,33]$ (symbols).

The appearance of each fission channel is linked to a specific minimum in the mass-asymmetry dependent potential close to the outer saddle. At this stage, as already explained above, we empirically determine the depths and the widths of the potential minima of the different fission channels at the outer saddle by the weights and the widths of the corresponding components in the empirical nuclide distributions. For this purpose, we need to calculate the number of states available in the different potential minima. This time, the Fermi-gas level density is not realistic: we have to consider the level density in a configuration with substantial shell effects. For this purpose, we use the analytical relation proposed by Ignatyuk et al. [36].

To determine the potential minima, we need the knowledge on the macroscopic and microscopic contributions to the potential. According to the previous discussion, we represent the macroscopic part by the parabolic potential deduced by Itkis et al. [35] from the widths of the mass distributions at high excitation energies.

In order to determine the microscopic contribution we consider the results of two-centre shell-model calculations $[25,37]$. They reveal that the shell effects at the outer barrier are qualitatively similar to the shells in the separate fragments. This would indicate that the structure of the wave functions is quite similar all the way from the outer saddle to scission. A similar conclusion was also obtained in ref. [26]. This is not any more valid for more compact shapes, since the energetically favoured shape at the inner saddle is triaxial and mass-symmetric. Based on the investigations of Wilkins et al. [31] on the scission-point configuration, we assume that the most important shells behind the Standard 1 fission channel are $N=82$ and $Z=50$, while the Standard 2 fission channel is related to the $N \approx 90$ strongly deformed shell. To quantitatively determine the shell effects in massasymmetry at the outer barrier, we consider the measured mass distribution of the fission fragments from ${ }^{238} \mathrm{U}(\mathrm{n}, \mathrm{f})[38,39]$. As we assume that the shell $N \approx 90$ is solely responsible for the appearance of the Standard 2 fission channel, its properties can be directly extracted form the observed Standard 2 channel. On the other hand, the properties of $N=82$ and $Z=50$ cannot be determined only from the data on the Standard 1 fission channel. Here, we make an assumption that the strength of the Standard 1 channel is given by the interplay of the $N=82$, $Z=50$ shells and the liquid-drop potential as a function of

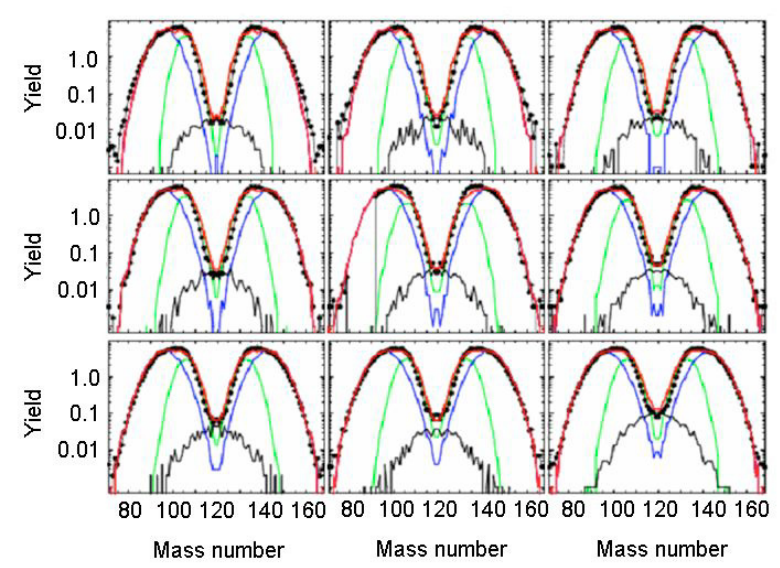

Fig. 2. Calculated mass distributions (red line) for neutron-induced fission of ${ }^{238} \mathrm{U}$ in comparison with experimental data (black symbols) $[38,39]$ for different values of the excitation energy above the fission saddle $(0.2,1.0,1.5,2.0,2.5,3.0,3.5,4.0$ and $6.0 \mathrm{MeV}$ going from the upper left corner) of the composite system ${ }^{239} \mathrm{U}$. The calculated individual contributions of the different fission channels are shown in addition: Standard 1 (green), standard 2 (blue), and superlong (black).

the $N / Z$ degree of freedom at the outer saddle. We introduce then one parameter as a common scaling factor for the total strength of the two shell effects relative to the empirical shell corrections in ground-state masses around ${ }^{132} \mathrm{Sn}$. In this way, we obtain simultaneously both the strength of the Standard 1 fission channel and the charge polarization at saddle. A similar procedure determines the charge polarization of this channel at scission.

As demonstrated in figure 2, taking all these ingredients one obtains a rather consistent description of the measured fission-fragment mass distribution from ${ }^{238} U(n, f)$, which reproduces the decrease of the relative population of the asymmetric fission channels with increasing excitation energy just by introducing the above-mentioned three shells corresponding to the Standard 1 and Standard 2 fission channels, and by considering the washing out of the shell effects with excitation energy [36]. From our adjusted parameters it appears that the spherical $N=82$ and $Z=50$ shells are considerably weaker than the shell effects we know from the ground-state masses around ${ }^{132} \mathrm{Sn}$. It can be speculated that the additional matter in the neck disturbs the symmetry of the nascent heavy fragment and reduces the shell gaps compared to the ideal spherical configuration we meet in ${ }^{132} \mathrm{Sn}$. The dominating appearance of the Standard 2 fission channel in ${ }^{238} \mathrm{U}(\mathrm{n}, \mathrm{f})$, see figure 2, seems to indicate that the deformed $N \approx 90$ shell, which according to the results of the shell-model calculations [27,31] appears less strong in the separate fragments, is less affected by the neck.

In order to calculate the $N / Z$ of the fragments at the scission point, we proceed in a similar way as in the case of high-energy fission. For the Standard 1 fission channel we consider an additional charge-polarization effect as explained above. After having adjusted the strengths and the widths of the three shells to the mass distributions of the system ${ }^{238} \mathrm{U}(\mathrm{n}, \mathrm{f})$, we are interested to check the predictive power of the model by applying it to other systems. We have 


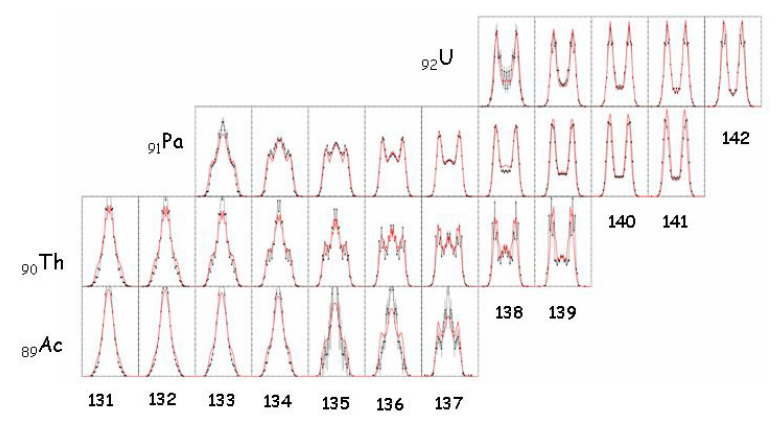

Fig. 3. Comparison between measured [2] (black dots) and calculated (red line) fission-fragment nuclear-charge distributions in the range $\mathrm{Z}=24$ to $\mathrm{Z}=65$ from electromagnetic-induced fission of secondary beams ranging from ${ }^{220} \mathrm{Ac}$ to ${ }^{234} \mathrm{U}$ shown on a chart of the nuclides.

chosen a set of data from ref. [2] on nuclear-charge distribution measured in electromagnetic-induced fission of several secondary beams ranging from ${ }^{220} \mathrm{Ac}$ to ${ }^{234} \mathrm{U}$, were we can observe a transition from a single-humped over a triplehumped to a double-humped nuclear-charge distribution. The good agreement with experimental data seen in figure 3 proves that a common description of ${ }^{239} \mathrm{U}$ and a series of secondary beams from ${ }^{220} \mathrm{Ac}$ to ${ }^{234} \mathrm{U}$ is possible by using the same set of parameters.

In fact, our model is able to reproduce the mass and element distributions over a large range of fissioning systems with only three shells present in the heavy fragment: $N=82$ and $Z=50$ for the Standard 1 fission channel, and $N \approx 90$ for the Standard 2 fission channel. Of course, it is possible that the features of these two fission channels are influenced by additional shells present in, e.g., the light fragment which we have not taken into account. The good agreement with a large set of experimental data seen in figures 2 and 3 supports the idea that the three above-mentioned shells are decisive in forming Standard 1 and Standard 2 fission channels. Moreover, these results would also suggest that the shell effects at the outer saddle have strong similarities with the shell effects in the separate fragments, thus representing empirical confirmation of previously performed theoretical works $[25,26,37]$.

\section{Conclusions}

We have formulated the most salient feature of our model as a rather peculiar application of the macroscopic-microscopic approach to nuclear properties. In our consideration of the properties of the fissioning system at the saddle configuration, we attribute the macroscopic properties to the strongly deformed fissioning system, while the microscopic properties are attributed to the qualitative features of the shell structure in the nascent fragments. This way, the macroscopic and the microscopic properties are strongly separated, and the number of free parameters is independent from the number of systems considered. This makes extrapolations in experimentally unexplored regions more reliable. With one and the same set of the model parameters we are able to reproduce a large variety of experimental data on mass and nuclear-charge distributions in low- and high-energy fission. Considering this success, we conclude that the present model has a remarkable predictive power, once the parameters have carefully been deduced from experimental fission-fragment distributions.

\section{References}

1. F.J. Gönnenwein, Radiation Effects 94, 205 (1986).

2. K.-H. Schmidt et al, Nucl. Phys. A 665, 221 (2000).

3. F. Rejmund, A.V. Ignatyuk, A.R. Junghans, K.H. Schmidt, Nucl. Phys. A 678, 215 (2000).

4. D. Hilscher, H. Rossner, Ann. Phys. Fr. 17, 471 (1992).

5. C. Schmitt et al. (accepted in Phys. Rev. Lett).

6. S. Ilevski et al., Phys. Rev. Lett. 92, 112502 (2004).

7. J.J. Cowan, F.-K. Thielemann, J.W. Truran, Phys. Rep. 208, 267 (1991).

8. Y. Kadi, A.Herrera-Martinez, Nucl. Instrum. Meth. A 562, 574 (2006).

9. G.H. Marcus, Prog. Nucl. Energy 37, 5 (2000).

10. J.L. Jones et al., Nucl. Instrum. Meth. A 562, 1085 (2006).

11. M.T. Kinlaw, A.W. Hunt, Nucl. Instrum. Meth. A 562, 1081 (2006).

12. P. Möller, D.G. Madland, A.J. Sierk, A. Iwamoto, Nature 409, 785 (2001).

13. H. Goutte, J.-F. Berger, P. Casoli, D. Gogny, Phys. Rev. C 71, 024316 (2005).

14. M.C. Duijvestijn, A.J. Koning, F.-J. Hambsch, Phys. Rev. C 64, 014607 (2001).

15. F. Atchison, Jül-Conf-34 KFA-Jülich, Germany (1980), p. 17.

16. V.A. Rubchenya, J. Äystö, Nucl. Phys. A 701, 127 (2002).

17. Z. Patyk et al., Nucl. Phys. A 491, 267 (1988).

18. J. Benlliure et al., Nucl. Phys. A 628, 458 (1998).

19. K. Kruglov et al., Eur. Phys. J. A 14, 365 (2002).

20. M.V. Ricciardi et al., Phys. Rev. C 73, 014607 (2006).

21. A. Keli, M.V. Ricciardi, K.-H. Schmidt (in preparation).

22. K.-H. Schmidt et al., Nucl. Phys. A 710, 157 (2002).

23. B. Jurado et al., Nucl. Phys. A 747, 14 (2005).

24. P. Nadtochy et al. (in preparation).

25. J. Maruhn, W. Greiner, Z. Phys. 251, 431 (1972).

26. V.V. Pashkevich, Nucl. Phys. A 477, 1 (1988).

27. I. Ragnarsson, R.K. Sheline, Phys. Scr. 29, 385 (1984).

28. Ya. Rusanov et al., Phys. At. Nucl. 60, 683 (1997).

29. P.N. Nadtochy, G.D. Adeev, A.V. Karpov, Phys. Rev. C 65, 064615 (2002).

30. P. Fong, Phys. Rev. C 17, 1731 (1978).

31. B.D. Wilkins et al., Phys. Rev. C 14, 1862 (1976).

32. M.G. Itkis et al., Phys. Part. Nucl. 29, 160 (1998).

33. J. Taieb et al., Nucl. Phys. A 724, 413 (2003); M. Bernas et al., Nucl. Phys. A 725, 213 (2003); M. Bernas et al., Nucl. Phys. A 765, 197 (2006).

34. P. Armbruster, Nucl. Phys. A 140, 385 (1970).

35. M.G. Itkis et al., Sov. J. Nucl. Phys. 43, 719 (1986).

36. A.V. Ignatyuk, G.N. Smirenkin, A.S. Tiskin, Yad. Fiz. 21, 485 (1975) [Sov. J. Nucl. Phys. 21, 255 (1975)].

37. A. Karpov (private communication).

38. F. Vives et al., Nucl. Phys. A 662, 63 (2000).

39. C.M. Zöller, Ph.D. thesis, Technical University Darmstadt, 1995. 